
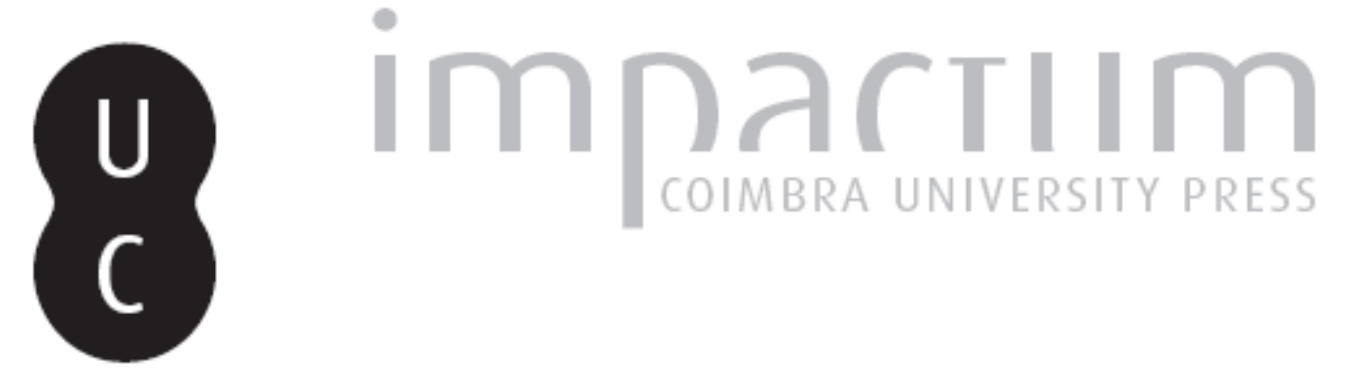

\title{
Posidonius source de Plutarque en matière d'éthique c'est-à-dire La théorie des passions psychiques chez Galien et Plutarque
}

\author{
Autor(es): $\quad$ Becchi, Francesco
}

Publicado por: International Plutarch Society

URL persistente:

URI:http://hdl.handle.net/10316.2/37653

DOI:

DOI:http://dx.doi.org/10.14195/0258-655X_8_2

Accessed : $\quad$ 26-Apr-2023 16:35:05

A navegação consulta e descarregamento dos títulos inseridos nas Bibliotecas Digitais UC Digitalis, UC Pombalina e UC Impactum, pressupõem a aceitação plena e sem reservas dos Termos e Condições de Uso destas Bibliotecas Digitais, disponíveis em https://digitalis.uc.pt/pt-pt/termos.

Conforme exposto nos referidos Termos e Condições de Uso, o descarregamento de títulos de acesso restrito requer uma licença válida de autorização devendo o utilizador aceder ao(s) documento(s) a partir de um endereço de IP da instituição detentora da supramencionada licença.

Ao utilizador é apenas permitido o descarregamento para uso pessoal, pelo que o emprego do(s) título(s) descarregado(s) para outro fim, designadamente comercial, carece de autorização do respetivo autor ou editor da obra.

Na medida em que todas as obras da UC Digitalis se encontram protegidas pelo Código do Direito de Autor e Direitos Conexos e demais legislação aplicável, toda a cópia, parcial ou total, deste documento, nos casos em que é legalmente admitida, deverá conter ou fazer-se acompanhar por este aviso.

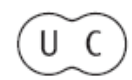




\title{
Posidonius source de Plutarque en matière d'éthique c'est-à-dire
}

\section{La théorie des passions psychiques chez Galien et Plutarque par Francesco Becchi \\ Università degli Studi di Firenze \\ francesco.becchi@unifi.it}

\begin{abstract}
The great revolution made by the most scientific of the Stoics, Posidonius, is the fact that passions cease to be perversions ( $\delta 1 \alpha \sigma \tau \rho o \varphi \alpha i)$ of reason. But the philosopher of Apamea not only recognizes the existence of passions as natural impulses that find their root in the body, but also admits the existence of psychic passions that acquire pathological character as a result of false judgments and empty opinions and that are the sign of a weakness ( $\dot{\alpha} \sigma \theta \dot{\varepsilon} v \varepsilon 1 \alpha$ vel $\dot{\alpha} \tau o v i ́ \alpha)$ of the soul. And it is this weakness of the soul the cause of the widespread passion that, in the early centuries of the vulgar era, authors with particular force, such as Plutarch and Galen, declare.
\end{abstract}

Key-Words: Psychopathology, Theory of passions, Passion and knowledge in Posidonius, Plutarch, and Galen

Dans une note, parue dans le volume «Les passions antiques et médiévales», publié sous la direction de Bernard Besnier, Pierre-François Moreau et Laurence Renault et édité par les Presses Universitaires de France dans le 2003, Jean-François Pradeau écrit : «Les anciens ne séparent pas ce qui relève de la "psychologie ", entendue comme analyse de la nature de l'âme, de ses facultés comme de ses affections, de ce qui relève par ailleurs de l'éthique, c'est-à-dire des moeurs

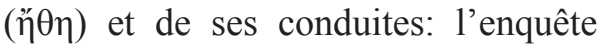
psychologique est l'hypothèse sur la quelle la thèse éthique doit être établie» ${ }^{1}$. En effet, de la doctrine psychologique de la nature de l'âme dépend non seulement la théorie des passions qui représent, selon les mots

1 Pradeau, p. 15.

Ploutarchos, n.s., 8 (2010/2011) 13-24

ISSN 0258-655X 
de Froidefond, «le moteur de l' âme» ${ }^{2}$, mais aussi celle des vertus et d'autres doctrines morales telles celle du bien suprême. Posidonius lui-même le confirme, selon les témoignages que nous fournit son admirateur Galien qui dans le De placitis Hippocratis et Platonis ecrit qu'il «n'est pas resté fidèle au radicalisme des premiers temps du stoïcisme», et qu'il «a infléchi le rigorisme intellectuel de Chrysippe vers des solutions plus modérées, plus ouvertes à l'expérience» ${ }^{3}$, en prenant ses distances du monisme psychologique et donc de la doctrine de la passion-jugement.

La critique que le plus scientifique des Stoïciens ${ }^{4}$ apporta au système psychologique et éthique de Chrysippe, si nous devons croire au témoignage de Galien, n'est pas en substance différente de celle qu'on lit dans le De virtute morali et dans les traités antistoïciens de Plutarque. Tous deux indiquent le monisme psycho- logique comme étant la cause de l'erreur de Chrysippe: la véritable origine des passions lui a échappé. Celles-ci ne viennent pas de l'extérieur de l'homme ${ }^{5}$, mais trouvent leur origine à l'intérieur de l'âme humaine ${ }^{6}$. En effet ce que reprochent essentiellement Posidonius et Plutarque à Chrysippe n'est pas seulement d'avoir nié l'évidence et de s'être contredit en reconnaissant en réalité ce qu'il nie dans ses écrits mais aussi et surtout de n'avoir pas su fornir une explication convaincante de l'étiologie de la passion, c'est-à- dire de n'avoir pas réussi à distinguer la cause de la $\pi \lambda \varepsilon 0-$

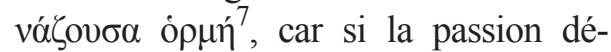
pendait de l'opinion et était la raison, celle-ci ne pourrait dépasser sa propre mesure (Gal., PHP IV 3. 1-3, p. 246248 De Lacy) et l'on ne pourrait expliquer le motif pour lequel la passion avec le temps s'apaise et cesse bien que l'opinion reste inchangée ${ }^{8}$.

2 Froidefond, p. 202.

3 GLiBERT-Thirri, p. 394.

4 Gal., Quod animi $11=$ IV 819 K.

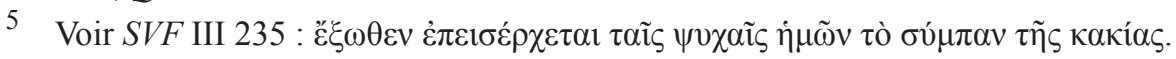

6 Sur les passions qui ne viennent pas de l'extérieur mais de l' intérieur voir aussi Plut., aud. 38C. Toutefois pour Plutarque à la différence de Posidonius (voir Gal., Quod animi mores IV 819 s. = Posid., Fr. 423 Theiler), la racine ( $\mathfrak{j}$ í $\alpha)$ de la méchanceté n'est pas représentée par les passions qui constituent le principe de l'action (Plut., an. corp. aff. $501 \mathrm{C}$ ), mais

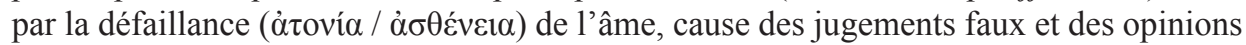
vaines, due à l'ả $\mu \alpha \theta i ́ \alpha$ et à l' $\alpha$ $\pi \alpha 1 \delta \varepsilon v \sigma i ́ \alpha$, qui sont en réalité le vrai mal du siècle.

7 Cf. Gal., PHP V 1. 5-6, p. 292 DE LACY.

8 Glibert-Thirry, p. 425: «Pour Posidonius, toutes les difficultés que présente la théorie chrysipéenne des passions tiennent à une erreur de méthodologie. C'est pour ne pas avoir posé la question de la cause du phénomène passionnel que Chrysippe a fait échouer son projet». 
C'est ainsi que la grande révolution accomplie à l'intérieur du Portique par Posidonius est celle-ci: les passions provoquées par la maladie de l'âme

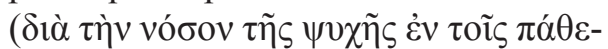

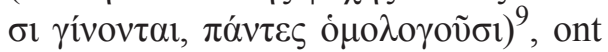
cessé de représenter des perversions

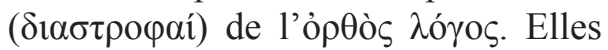
ne constituent pas un phenomène qui

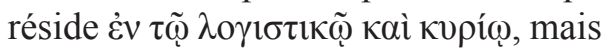

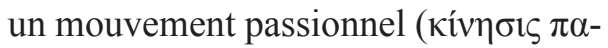

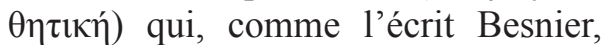
«n'est pas déliberé ni choisi» ${ }^{10}$, mais provient des facultés passionnelles

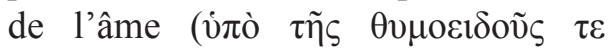

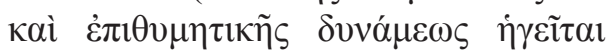

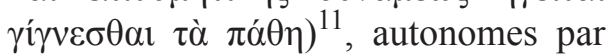

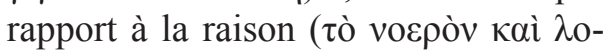
$\gamma(\sigma \tau \imath \kappa o ́ v)$ et intimément liées au corps par une nécessité naturelle ${ }^{12}$.

Mais bien que le philosophe d'Apaméee et l'intellectuel de Chéronée considèrent la passion comme un mou-

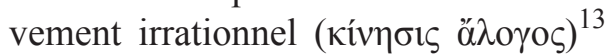
ou une impulsion violente et excessive

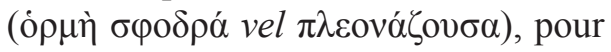

ceux-ci toutefois elle n'est plus irrationnelle dans l'acception stoïcienne de «contraire à la droite raison» et elle assume la valeur académico-péripatéticienne de «provenant de la faculté irrationnelle de l'âme», que témoigne aussi le péripateticien Aspasius presque contemporain de Plutarque dans son commentaire à l'Éthique à Nicomaque d'Aristote ${ }^{14}$.

En prenant parti pour Pythagore, Platon et Aristote contre le monisme des Stoïciens, Posidonius et Plutarque marquent un retour à la théorie des ápxaior et excluent d'une part que la

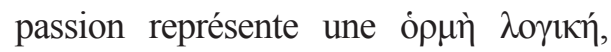
c'est-à-dire, une impulsion rationnelle, tendance qui se trouve seulement chez un animal doué de $\log _{0}{ }^{15}$, et d'autre part la possibilité pour l'être humain d'une vie parfaitement divine, capable d'oublier les nécessités de l'humaine condition dans l'apathie totale, qui représente un idéal divin ou bestial ${ }^{16}$, de toutes façons étranger à la nature humaine ${ }^{17}$.

9 Gal., PHP IV 5. 29s., p. 264s. DE LACY.

10 Voir Plut., virt. mor. 450A.

11 Gal., PHP IV 3. 3, p. 248 DE LACY.

12 Cf. Plut., virt. mor. 450E. Sur l'existence d'une irrationalité plongée dans le corps voir Sassi (1992), p. 362 s.

13 Cf. Plut., cons. ad uxor. 609A (

14 Asp., EN, CAG XIX. 1, ed. G. Heylbut, Berolini 1889, p. 35. 16s.

15 Gal., PHP V 1. 10, p. 294 De Lacy. Voir Besnier, p. 30, 2.

16 Plut., Public. 6, 5.

17 Glibert-Thirry, p. 434: «L'idéal d'apathie au sens où l'entendait Chrysippe s'avère, nous semble-t-il, irréalisable dans la théorie posidonienne». 


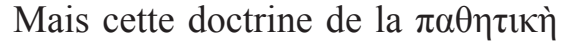

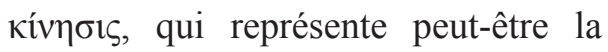
question la plus délicate de toute la théorie morale posidonienne, tourne autour d'un passage du $\mathrm{V}$ livre du De placitis de Galien, interprété de différentes façons. Voici le texte de ce passage que je rapporte dans la forme transmise par la tradition manuscrite avec la nécessaire intégration de Müller ( $\gamma i ́ v \varepsilon \sigma \theta \alpha \imath)$ :

Gal., PHP V 5. 21, p. 320 De Lacy $=$ Posid., Fr. 416, p. 337.3 Theiler

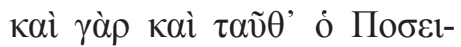

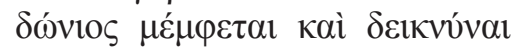
$\pi \varepsilon 1 \rho \tilde{\alpha} \tau \alpha 1 \quad \pi \alpha \sigma \tilde{\omega} \nu \quad \tau \tilde{\omega} \nu \quad \psi \varepsilon v \delta \tilde{\omega} \nu$

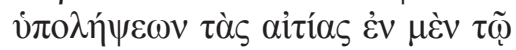

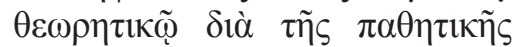
ó $\lambda \kappa \tilde{\eta} \varsigma<\gamma^{\prime} \dot{v} \varepsilon \sigma \theta \alpha l>, \pi \rho 0 \eta \gamma \varepsilon \tilde{i} \sigma \theta \alpha l$

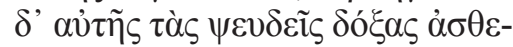

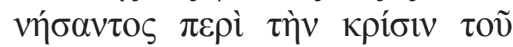

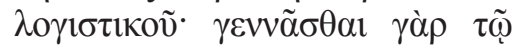

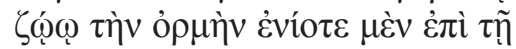

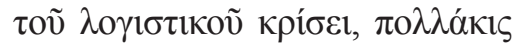

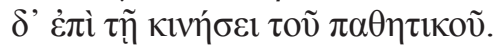

5 post $\theta \varepsilon \omega \rho \eta \tau \imath \kappa \tilde{a}$ suppl. $\sigma \kappa \varepsilon \dot{\mu} \mu-$

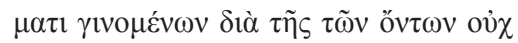

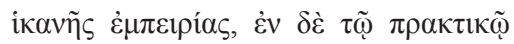

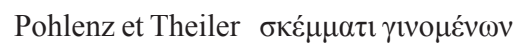

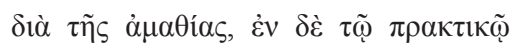

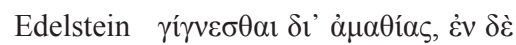
$\tau \tilde{\varphi} \pi \rho \alpha \kappa \tau \iota \kappa \tilde{~ D e ~ L a c y ~} 6$ post ó $\lambda \kappa \tilde{\eta} \varsigma$

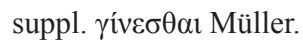

Fillion-Lahille, p. 156.

19 GiLl, p. 151.

20 Voir Glibert-Thirry, p. 432.
Posidonius critique aussi ces théories et essaie de démontrer que les causes de tous les faux jugements résident dans le theoretikon par suite d'un entraînement issu du pathetikon, mais que les opinions erronées précèdent cet entraînement en raison de la faiblesse du logistikon en ce qui concerne la faculté de juger; car la tendance chez l'être vivant résulte quelquefois d'un jugement du logistikon, et souvent de l'impulsion du pathetikon.

Ce passage a été «généralement considéré comme obscur et fort corrompu» ${ }^{18}$, si bien que «la plupart des éditeurs modernes» (Müller, EdelsteinKidd, Theiler, De Lacy) dans le sillage de Pohlenz ont pensé à une lacune plus ou moins grande ${ }^{19}$. Mais si le texte est apparu obscur dans la forme trasmise par la tradition manuscrite en raison des difficultés à expliquer les conditions pour lesquelles les causes des opinions erronées résident dans la faculté rationnelle par effet de l'entraînement affectif $(\pi \alpha \theta \eta \tau \tau$ in ò $\lambda \kappa \eta ́)$, il ne résulte pas pour autant plus clair avec les intégrations proposées par les différents éditeurs de Galien et de Posidonius. En effet, en admettant que la faculté passionnelle quand elle est forte, détermine le rationnel de sorte que les jugements finissent par s'accorder avec les mouvements passionnels et les dirigentes ${ }^{20}$, ceux-ci confirment le 
principe contraire à la conception de Posidonius $^{21}$ que l'inférieur puisse corrompre le supérieur ${ }^{22}$.

Enfin en 1984 ce texte, si contesté et manipulé par nombre de chercheurs éminents et remarquables pour n'aboutir malgré tout qu'à une interprétation assez confuse et embarrassée, a semblé, pour Janine Fillion-Lahille, présenter «un sens parfaitement logique» et «se suffire à lui même»» ${ }^{23}$.

Mais meme l'exégèse de la savante française-pour la quelle Posidonius n'est pas sorti des limites fondamentales du stoïcisme et l'impression de rupture avec le rationalisme stoïcien traditionnel «n'est due qu'à l'interprétation tendancieuse à travers laquelle sa doctrine des passions nous a été transmise par Galien $»^{24}$ tombe dans les mêmes contradictions: la passion pour Posidonius resterait comme pour Chrysippe un jugement de notre raison $^{25}$, accompli par un logistikon défaillant, frappé par un entraînement

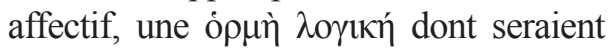
exclus les enfants qui ne sont pas encore rationnels et les animaux qui ne le sont pas, non plus. Ainsi le mouvement affectif consisterait «en une conviction qui s'est développée à tort ( $\psi \varepsilon v \delta \grave{c}$

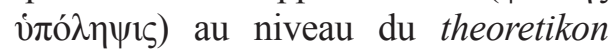
( $\dot{\varepsilon} \vee \tau \tilde{\omega} \theta \varepsilon \omega \rho \eta \tau \imath \kappa \tilde{\omega})$, prenant ainsi les proportions d'une force irrationelle qui nous entraîne irrésistiblement $(\pi \alpha-$

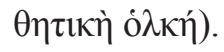

Plus récemment, en 2003, dans ce débat est intervenu Cristopher Gill qui défend le texte transmis par la tradition et dans le sillage de l'exégèse de FillionLahille croit lui aussi que Galien ne trasmet pas fidèlement la théorie posidonienne: en effet selon Galien, en refusant le modèle chrysippeen de l'âme unifiée et en adoptant la psychologie platonicienne, Posidonius n'accepte pas que la passion soit rationelle quant à sa fonction. Pour Gill - qui reconnaît comme seule innovation importante sur ce sujet...l'idée des «mouvements affectifs» (pathêtikai kinêseis), qui ne sont pas des passions, mais des réactions naturelles - Posidonius reste en psychologie un stoïcien qui modifie et compléte la théorie chrysippéenne des passions, mais ne la remplace pas. En conclusion selon Gill dans le passage important en question du De placitis le philosophe d'Apamée

\section{${ }^{21}$}

22

Cf. Gal., PHP V 6. 23-24, p. 330 De LaCY = Posid., Fr. 162 E.-K.

Pour Glibert-Thirry (p. 432-33) aussi, qui suit le texte corrigé par Edelstein, le comportement passionnel se manifesterait quand «la raison est faible et ne peut résister à l'inclination du pathèticon».

23 Fillion-Lahille, p. 158.

24 Fillion-Lahille, pp. 153-154.

25 Fillion-Lahille, p. 156.

Ploutarchos, n.s., 8 (2010/2011) 13-24

ISSN 0258-655X 
marquerait une distinction entre les impulsions qui, dérivées d'un jugement rationnel, surviennent chez les adultes humains, en tant que êtres rationnels, et celles qui touchent les enfants et les animaux $^{26}$, chez lesquels le logistikon est soit inexistant soit encore impropre à assumer son rôle.

Cette exégèse repose sur une interprétation arbitraire, reprise par FillionLahille, selon laquelle le processus affectif est fondé sur un jugement du

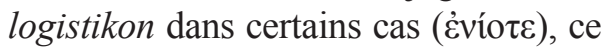
serait à dire, «chez l'homme adulte»,

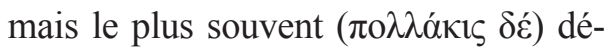
rive de l'impulsion du pathetikon chez les êtres denués de raison comme les enfants et les animaux.

Quoi qu'il en soit, en affirmant que «selon Posidonius nous devons aussi supposer qu'il y a des mouvements affectifs pour expliquer pourquoi nous avons des passions ${ }^{27}$, Gill semble reconnaître en quelque manière l'impossibilité d'expliquer chez Posidonius le phenomène passionnel seulement sur une base rationnelle avec une rupture entre les adultes humains d'un coté et les enfants et les bêtes de l'autre. En effet Posidonius encore avant Plutarque «eut honte d'adopter la théorie de toute évidence fausse des autres stoïciens»

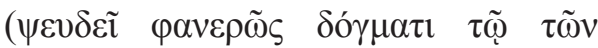
$\ddot{\alpha} \lambda \lambda \omega v \Sigma \tau \omega \ddot{\kappa} \tilde{\omega} v)$, arrivés à un tel point de polémique qu'ils niaient les passions aux enfants, qui bien sur ne sont pas encore des êtres rationnels, et aux animaux, êtres irrationnels ${ }^{28}$.

Pour Posidonius, la démonstration de l'indépendance des passions de la raison chez l'adulte, se fonde précisément sur l'évidence que les mêmes passions existent chez des êtres non encore rationnels ou, selon les Stoïciens, dépourvus de rationalité ${ }^{29}$. Par conséquent, si la thèse qui situe le mouvement affectif dans un modèle psychologique essentiellement chrysippéen était vraie, Posidonius aurait dû soit nier l'existence de la passion chez

26 GiLL, p. 151.

27 GiLL, p. 150.

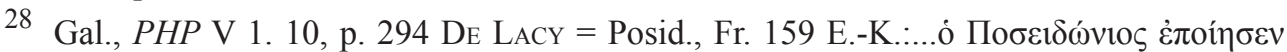

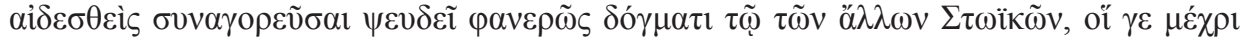

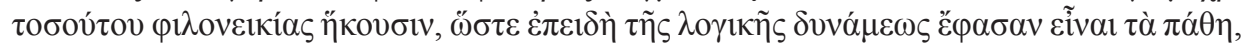

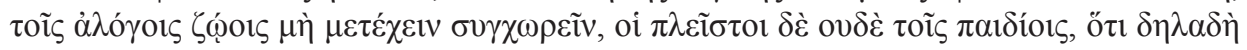

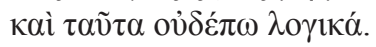

29 Gal., PHP V 5. 1s., p. 316s. DE LACY. Les textes ne confirment pas la thèse avancée avec réserve par Gill (p. 150-152), selon laquelle l'innovation posidonienne consisterait en une rupture entre la passion rationnelle des adultes et celle des enfants. En ceci, toutefois Gill se détache de la thèse de Fillion-Lahille (p. 159), pour qui Posidonius nierait «l'existence de la passion chez les êtres non doués de raison». 
les animaux et les enfants, soit admettre pour la passion comme le fait Chrysippe pour la rationalité une rupture nette, que les textes ne confirment pas, entre les enfants et les animaux d'un côté et les adultes, de l'autre ${ }^{30}$.

À l'état actuel des études, deux interprétations de la doctrine posidonienne de la passion s'affrontent: celle de Galien qui en fait un mouvement de la faculté passionnelle et celle de Fillion-Lahille et maintenant de Gill aussi qui en font un jugement de la raison. Placé devant cette alternative, Pigeaud écrivait avec ironie : «Je pense que Galien...a raison contre Mme Fillion» ${ }^{31}$ et j'ajouterais même contre Gill. Je partage ce choix, même si je suis convaincu qu'il y a un fond de vérité dans chacune des deux thèses. La preuve n'en est pas seulement le fragment en question du De placitis, mais aussi un témoignage de Plutarque dont l'interprétation vulgate a fini par constituer la contre-épreuve de la justesse de la thèse rationaliste et par démontrer, comme l'écrit Michel Spanneut, que «Posidonius n'écarte pas pour autant du procès passionnel l'intervention de la raison» ${ }^{32}$.

Si l'on admet que Posidonius est resté plus orthodoxe qu'il apparaît à travers Galien et qu'en définitive il n'est pas cet anti-Chrysippe que l'intellectuel de Pergame nous dépeint, les textes montrent que, au moins en

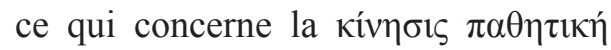
l'élève de Panetius a préferé se rendre

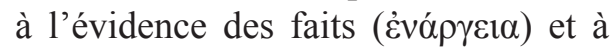
l'expérience plutôt qu'au rationalisme systématique de Chrysippe ${ }^{33}$.

Toutefois une exégèse correcte du fragment du De placitis ne peut faire abstraction du contexte dans le quel Galien fait part des critiques apportées par Posidonius à la théorie chrysippéenne

30 Voir Fillion-Lahille (p. 159): «Bien que ce point (i.e.: la passion est un jugement) nous paraisse désormais suffisamment établi, l'on peut pourtant encore le soumettre à une contre-épreuve décisive. Posidonius admet-il l'existence de la passion chez les êtres non doués de raison ? Si la réponse était oui, notre thèse s'en trouverait incontestablement infirmée». Sur ce point voir Gill (p. 149) qui a montré que: «La seule innovation importante de Posidonius sur ce sujet est, je crois, l'idée que les adultes humains (comme les enfants humains et les bêtes) font l'expérience des «mouvements affectifs» (pathêtikai kinêseis) c'est-à-dire, des réponses affectives aux impressions».

31 Spanneut, p. 4676 n. 142.

32 Spanneut, p. 4676 n. 142 : «Plutarque dit expressément que Posidonius place "désirs, craintes et colères" “ dans les jugements et conceptions » (Fgt. EK 154, 13)».

33 Cf. Glibert-Thirry, p. 429: «L'expérience montre aussi que des facteurs d'ordre phisiologique et physique déterminent l'irrationel de l'âme et ses impulsions. "Les mouvements passionnels de l'âme suivent toujours la disposition du corps... ». Cf. Gal., PHP V 443, p. 322 De LaCY = Posid., Fr. 153 E.-K. 
des óp $\mu \alpha i^{34}$ et de l'origine du mal ( $\dot{\eta} \kappa \alpha \tau \grave{\alpha}$

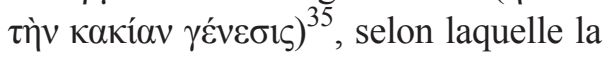
tendance naturelle à rechercher le plaisir et à fuir la douleur dépendrait même

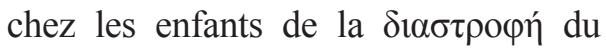
$\lambda$ ó $\gamma$ s, due au continuel apprentissage de la part du milieu extérieur ( $\dot{\varepsilon} \kappa$ $\kappa \alpha \tau \eta \chi \eta ́ \sigma \varepsilon \omega \varsigma \tau \tilde{\omega} v \pi \circ \lambda \lambda \tilde{\omega} v \dot{\alpha} v \theta \rho \omega ́ \pi \omega v)$ et à la nature même des choses $(\dot{\varepsilon} \xi \alpha \hat{\jmath} \tau \tilde{\eta} \varsigma$

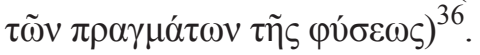

Contrairement à cette doctrine de Chrysippe pour laquelle l'ensemble du mal parvient à l'âme de l'extérieur ${ }^{37}$ entrainé par des opinions erronées ${ }^{38}$

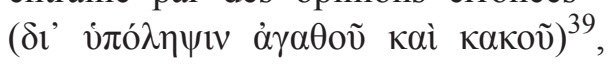
Posidonius essaie de démontrer que la passion a des causes intérieures et dans le passage en question il semble accomplir une nette distinction des rôles entre la faculté théorétique

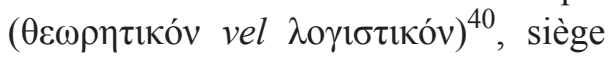
de fausses opinions, et la faculté passionnelle, siège des passions. En effet, pour Posidonius, les jugements et les opinions appartiennent à la faculté rationnelle, alors que les impulsions passionnelles, qui dépendent de la constitution physique, sont le propre des facultés passionnelles de l'âme ${ }^{41}$.

Si c'est là le scénario où se place notre passage, en pensant à un saut par omoteleut je suggérerais exempli gratia d'intégrer après $\theta \varepsilon \omega \rho \eta \tau \iota \kappa \tilde{\omega}<\gamma$ <

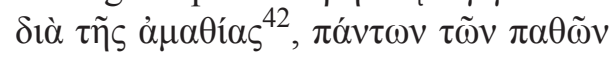

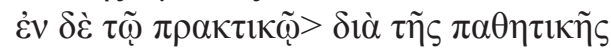
ó $\mu \tilde{\eta} \bar{\zeta}$, en remplaçant le terme transmis ò $\lambda \kappa \tilde{n} \varsigma$, inusité au moins au niveau éthique, par le terme ópuñs. Voici donc le sens du passage : Posidonius essaie de démontrer que les causes de tous les jugements faux se situent dans le theoretikon par suite de l'ignorance mais que celles de toutes les passions se trouvent dans le praktikon par suite d'une impulsion passionnelle.
34

35

36

37

38

39

40

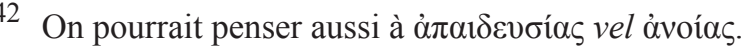


Dans ce cas, le philosophe d'Apamée n'opposerait pas l'erreur théorique à l'erreur pratique comme le pensait Pohlenz, ni la passion rationnelle des adultes aux mouvements affectifs des enfants et des animaux, mais l'activité rationnelle à l'activité passionnelle, admettant toutefois, en stoïcien, que parfois, quand la faculté rationnelle est faible, l'impulsion rationnelle précède l'impulsion passionnelle sans laquelle toutefois l'opinion fausse ou le faux jugement $^{43}$, qui dépendent de l' $\alpha \sigma \theta \varepsilon ́ v \varepsilon 1 \alpha$ et de l' à $\tau o v i ́ \alpha$ de l'âme, ne seraient à eux seuls des phénomènes suffisants pour déterminer la genèse de la passion ${ }^{44}$.

Cette interprétation a entre autres l'avantage d'être confirmée dans le $D e$ libidine et aegritudine de Plutarque ${ }^{45}$ qui attribue à Posidonius une subdivision des passions entre «psychiques» et

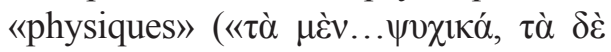

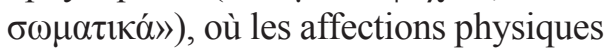
se distinguent en affections uniquement physiques comme les accès de fièvre, les frissons provoqués par le froid, les contractions et les dilatations, toutes manifestations liées à la constitution physique, et en affections physiques reliées à l'âme telles la léthargie ou la tristesse; de façon analogue les passions psychiques se divisent en passions uniquement ou absolument psychiques ( $\psi v \chi 1 \kappa \alpha ̀ ~ \mu \varepsilon ̀ v ~ \dot{\alpha} \pi \lambda \tilde{\omega} \varsigma)$, liées aux jugements et aux opinions ( $\tau \grave{\alpha} \dot{\varepsilon} v$

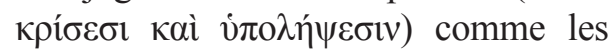
désirs, la peur, les sursauts de colère, et en passions psychosomatiques ( $\pi \varepsilon p$ ì $\sigma \tilde{\omega} \mu \alpha \psi \psi v \chi(\kappa \alpha ́)$ comme le tremblement, la pâleur, les changements d'aspect dus à la peur ou à la douleur ${ }^{46}$.

Ainsi sans renier l'indissolubilité de l'âme et du corps, qui forment la nature humaine, Plutarque semble distinguer dans son système éthique les passions, impulsions affectives ( $\alpha i$

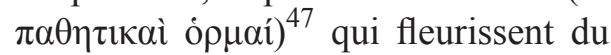
corps comme d'une racine et s'impo-

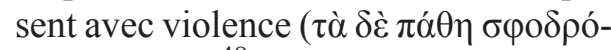
$\tau \eta \tau \varepsilon \zeta \dot{o} \rho \mu \tilde{\omega} v)^{48}$ en raison de leur nature irrationnelle $(s c .<\dot{0} \rho \mu \grave{\eta}>\tau \tilde{\omega}$ à $\lambda \hat{\gamma} \gamma \omega$

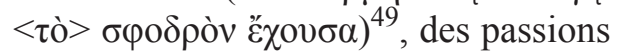

43

Gal., PHP V 5. 21, p. 320 DE LACY.

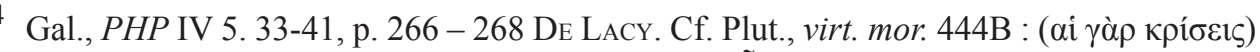

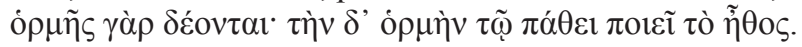

45 Plut., lib. et aegr. c. 6.

46 Plutarque, pour le quel l'homme constitue une unité indissoluble formée du corps et d'âme, considère les tremblements, les pâleurs, les changements d'aspect dus à la peur ou à la douleur, comme des conséquences à niveau physique des passions. Cf. Plut., virt. mor. 449A.

47 Plut., virt. mor. 451A.

48 Plut., an. corp. affect. 501C.

49 Plut., lib. et aegr. c 7.

Ploutarchos, n.s., 8 (2010/2011) 13-24

ISSN 0258-655X 


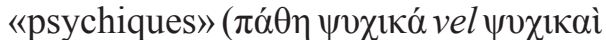
vóбol $)^{50}$ qui se manifestent violemment, entrainées par un jugement

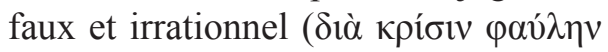

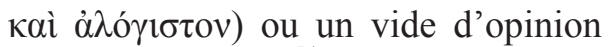

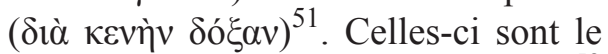

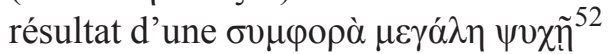
ou d'une faiblesse de l'âme (ג் / àtovía $)^{53}$, qui proie d'opinions erronées, laisse couler la passion comme $\delta$ ó $\xi \alpha \dot{\varepsilon} \mu \pi \alpha \theta \eta \varsigma^{54}$. Quand à un sentiment naturel qui est peu de chose ( $\mu$

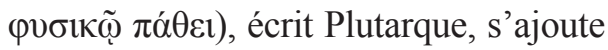
la conviction liée à une opinion erronée, la passion s'enflamme ( $\mu \alpha v i \kappa o ́ v)^{55}$, se laissant transporter outre mesure ${ }^{56}$. Or amplifier les passions, écrit même le Pseudo-Plutarque dans la Consolatio ad Apollonium est contraire à la nature et dérive d'une opinion erronée ( $\varphi \propto v \dot{\lambda} \eta$ $\delta o ́ \xi \alpha$ ) qui réside en nous ${ }^{57}$. Ainsi, ces passions, liées à l'ố $\gamma v 0 \alpha^{58}$ et dues

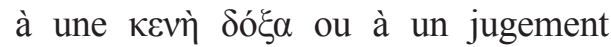

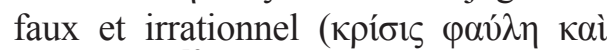
$\dot{\alpha} \lambda o^{\prime}(\sigma \tau \circ \varsigma)^{59}$, qui obsèdent et humilient l'âme, représentent pour le philosophe de Cheronée les pires maladies de l'âme

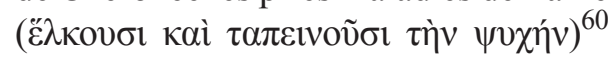
dont l'organe le premier compromis est celui-là même du jugement qui devrait

50 Plut., an. corp. affect. 500E-501A.

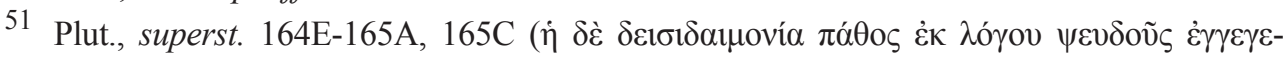

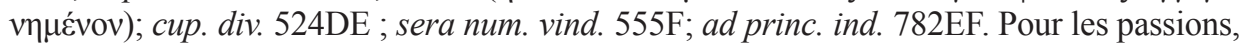

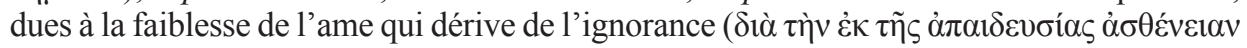

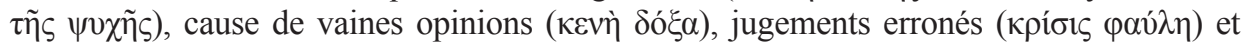

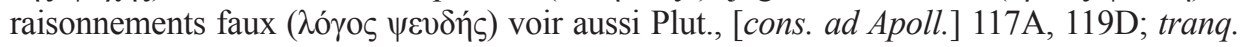
an. 465D, 468CD, 475D; gen. Socr. 584E ; exil. 600E, 602B ; cons. ad uxor. 609EF.

53.

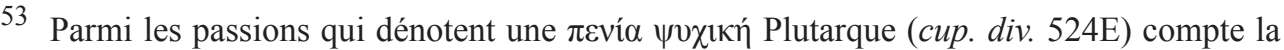

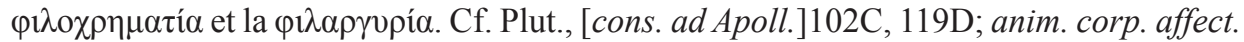
500EF; gen. Socr. 579F.

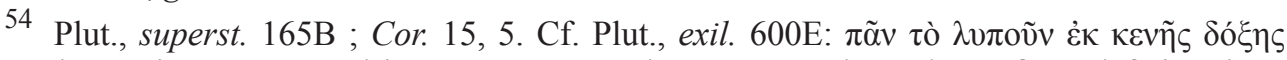

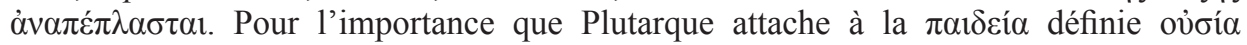

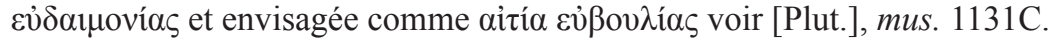

Plut., cons. ad uxor. 609EF.

56 Cf. Plut., [cons. ad Apoll.], 114D.

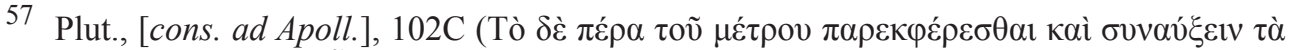

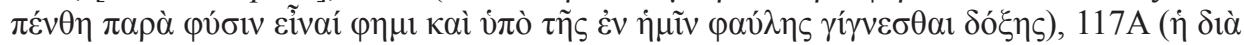

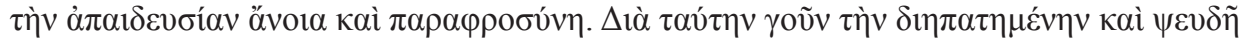

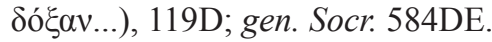

58 Plut., superst. 167AB.

59 Plut., cup. div. 524CD.

60 Plut., gen. Socr. 584E. 
au contraire constituer le premier soin et le premier remède de la passion $(\pi \rho \tilde{\omega} \tau o v$

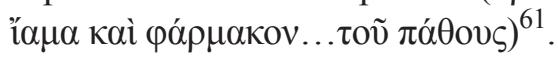

En concluant, je me demande et je Vous demande s'il est possible que cette distinction entre les passions les unes psy-

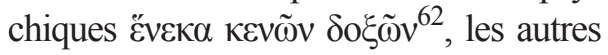
psycho-somatiques, arrive chez Plutarque de Posidonius. Je n'exclurai pas qu' en formulant la théorie des passion psychiques, où les jugements erronés et les opinions inconsistantes compromettent un

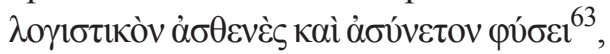
le philosophe de Chéronée en quelque manière ait eu présente à l'esprit la théorie posidonienne des passions.

\section{BibLIOGRAPHIE}

BESNIER, B.,

- "Aristote et les passions", dans BeSNIER Moreau - Renault (eds.), 2003, p. 30, 2.

Besnier, B. - Moreau, P. F.- Renault, L. (eds.), - Les passions antiques et médiévales, Paris, 2003.
Fillion-LahilLe, J.,

- Le De ira de Sénèque et la philosophie stoïcienne des passions, Paris, 1984.

FoIDEFond, CH.,

- "Plutarque et le platonisme", $A N R W$ II 36. 1, Berlin-New York, 1987, pp. 201-211.

GILL, CHR.,

- "Galien a-t-il compris la théorie stoicienne des passions ?", dans BESNIER - Moreau - Renault (eds.), 2003, pp. 145-152.

GLIBERT THIRRY, A.,

- "La théorie stoïcienne de la passion chez Chrysippe et son évolution chez Posidonius", Revue philosophique de Louvain, 75 (1977) 394.

Pradeau, J.-F.,

- "Platon, avant l'érection de la passion", dans Besnier - Moreau - Renault (eds.), 2003, p. 15.

SASSI, M. M.,

- "Plutarco antifisiognomico, ovvero: del dominio della passione", dans Gallo (ed.), 1992, pp. 362s.

Spanneut, M.,

- "Apatheia ancienne, apatheia chrétienne", dans $A N R W$ II 36. 7, Berlin-New York, 1994, p. 4676 n. 142.

61 Plut., garr. 510D.

62 Plut., gen. Socr. 584E.

63 Gal., PHP V 5. 28-29, p. 322 De LACY = Posid., Fr. 416, p. 337.3 TheILER. 